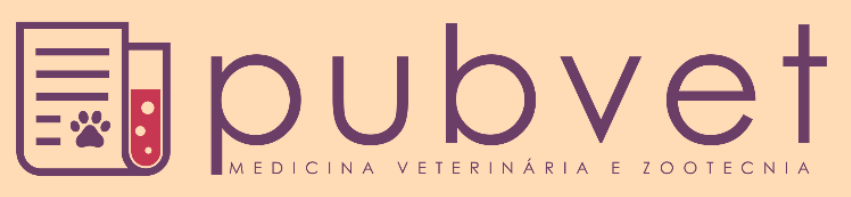

https://doi.org/10.31533/pubvet.v13n6a350.1-5

\title{
Enterectomia na correção de intussuscepção associada a linfoma alimentar obstrutivo em um cão jovem: Relato de caso
}

\author{
Patrícia Silva Vives $^{1 *}$, Marina Zanin ${ }^{2}$, Tainá Ança Evaristo ${ }^{3}$, Francisco de Assis Araújo

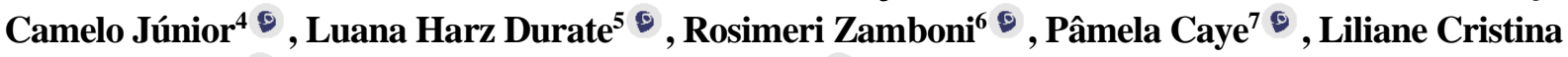 \\ Dias Jerônimo $^{7 \bullet}$, Josaine Cristina da silva Rappeti ${ }^{8}$, Fabrício de Vargas Arigony Braga ${ }^{8}$ \\ ${ }^{1}$ Médica Veterinária, Doutora em Cirúrgica e Clínica de Pequenos Animais, Técnica em Educação no Hospital de Clínicas \\ Veterinárias, Universidade Federal de Pelotas (UFPel), Pelotas Rio Grande do Sul, Brasil. \\ ${ }_{2}^{2}$ Médica Veterinária, Pós-graduanda em Ciências Fisiológicas - Universidade Federal do Rio Grande. Pelotas-RS, Br. \\ ${ }^{3}$ Discente em Medicina Veterinária, UFPel, Pelotas, RS, Br. ${ }^{4}$ Médico Veterinário, UFPel, Pelotas, $R S, B r$. \\ ${ }^{5}$ Médica Veterinária, Especialista em Imagenologia, UFPel, Pelotas, $R S, B r$. \\ ${ }^{6}$ Médica Veterinária, Mestre em Ciências Veterinárias, UFPel, Pelotas, RS-Br. \\ ${ }^{7}$ Médica Veterinária, Residente em Clínica Cirúrgica de Animais de Companhia, UFPel, Pelotas-RS, Br. \\ ${ }^{8}$ Docente do curso de Medicina Veterinária, Departamento de Clínicas Veterinárias, UFPel, Pelotas-RS, Br. \\ *Autor para correspondência, E-mail: patvivesvet@hotmail.com
}

Resumo. Linfoma é a neoplasia linfo-hematopoiética mais frequente em cães, e uma das mais quimiorresponsivas na medicina veterinária, portanto o tratamento geralmente é baseado em protocolos quimioterápicos, associados ou não, a ressecção cirúrgica. Na forma alimentar, é pouco comum em cães e caracteriza-se pela localização no trato gastrointestinal e/ou linfonodos mesentéricos e aqueles não tratados vivem entre quatro a seis semanas após o início dos sinais clínicos ou diagnóstico. Este trabalho tem como objetivo relatar o tratamento cirúrgico de um cão jovem com linfoma intestinal obstrutivo, que após enterectomia do segmento acometido, obteve diagnóstico histopatológico. Mesmo com a prescrição de quimioterapia, o tutor optou pelo tratamento de suporte apenas e o paciente teve sobrevida de oito semanas.

Palavras chave: neoplasia, diarreia, prolapso intestinal

\section{Enterectomy in the correction of intussusception associated with obstructive food lymphoma in a young dog: Case report}

\begin{abstract}
Lymphoma is the most frequent lymphohematopoietic neoplasia in dogs, and one of the most chemorresponsive in veterinary medicine, so the treatment is usually based onchemotherapy protocols associated or not to surgical resection. In food form, it is uncommon in dogs and characterized by the location in the gastrointestinal tract and mesenteric lymph nodes, and untreated live between four and six weeks after the start of clinical signals or diagnosis. This work aims to report the surgical treatment of a young dog with intestinal obstructive food lymphoma that, after an enterectomy of the affected segment, obtained diagnosis in the histopathological. Even with the prescription of chemotherapy, the tutor chose only for support treatment and the patient had an eight-week survival.
\end{abstract}

Keywords: neoplasia, diarrhea, intestinal prolapse 


\title{
Enterectomia para corrección de la intususcepción asociada al linfoma alimentario obstructivo en perro joven: Reporte de un caso
}

\begin{abstract}
Resumen. Linfoma es la neoplasia linfohematopoyética más frecuente en perros y una de las que más responden a la quimioterapia en medicina veterinaria, por lo que el tratamiento generalmente se basa en protocolos de quimioterapia asociados o no a la resección quirúrgica. En la forma alimentaria, es poco común en los perros y se caracteriza por la localización en el tracto gastrointestinal y ganglios mesentéricos, aquellos no tratados viven entre cuatro y seis semanas después del inicio de los signos clínicos o el diagnóstico. El objetivo de este trabajo es informar el tratamiento quirúrgico de un perro joven con linfoma intestinal obstructivo que, después de una enterectomia del segmento afectado, obtiene el diagnóstico histopatológico. Incluso con la prescripción de quimioterapia, el tutor eligió solo el tratamiento de soporte y el paciente tuvo una supervivencia de ocho semanas.
\end{abstract}

Palabras clave: neoplasia, diarrea, prolapso intestinal

\section{Introdução}

O linfoma é uma neoplasia originada em órgãos linfo-hematopoiéticos como baço, linfonodos, fígados e agregados linfoides (Vail et al., 2001). Normalmente é classificado de acordo com a localização anatômica, podendo ser denominado multicêntrico, intestinal ou alimentar, cutâneo e extranodular (Sequeira et al., 1999).

O linfoma alimentar é incomum e representa cerca de 5 a $7 \%$ de todos os linfomas que acometem o cão, sendo a segunda forma mais descrita nesta espécie (Vail et al., 2001). Se distribui amplamente ou em agregados no epitélio, lâmina própria e submucosa do intestino (Silva et al., 2003) e caracteriza-se pelo aparecimento como fenômeno primário no trato gastrointestinal, embora massas nesse local possam aparecer como extensão de outras formas de linfoma (Lowe, 2004).

A etiologia do linfoma canino vem sendo investigada, diferentemente do linfoma em felinos e bovinos, não é induzido pela infecção prévia por vírus (Fighera, 2002). Além disso, enquanto cães não há discussão sobre fatores de risco, tratando-se de gatos, sabe-se que aqueles positivos para FIV/FELV e/ou expostos à fumaça de cigarro são mais predispostos (Gieger, 2001).

De acordo com estudo retrospectivo realizado na Universidade Federal de Santa Maria, observou-se há tendência dessa neoplasia acometer cães idosos (Fighera et al., 2006), e machos, embora o linfoma de maneira geral não apresente predileção por sexo (Ettinger, 2003). Cardoso et al. (2004) citam apatia, perda de peso, vômito, diarreia e melena como sinais clínicos comuns em pacientes acometidos por linfoma alimentar. O diagnóstico é baseado no histórico, exames laboratoriais e de imagem, porém a confirmação é por meio da análise citológica e histopatológica (Vail \& Ogilvie, 2003).

Como tratamento, considera-se a associação de ressecção cirúrgica e quimioterapia, visto que essa neoplasia é considerada bastante quimiorresponsiva. Enquanto cães não tratados vivem, em média, quatro a seis semanas, 60 a $90 \%$ daqueles submetidos a quimioterapia apresentam remissão completa da doença. Embora a quimioterapia seja a primeira alternativa indicada nos casos de linfoma, em casos do subtipo alimentar pode ser considerada previamente a cirurgia, tendo em vista o prejuízo ao trânsito intestinal que a massa pode causar (Ettinger, 2003; Lowe, 2004).

O presente trabalho tem como objetivo relatar a ocorrência de intussuscepção em um cão jovem com diagnóstico de linfoma alimentar tratado unicamente por meio de enterectomia.

\section{Relato de caso}

Um cão, macho, quatro anos, sem raça definida e 27 quilos de massa corpórea, atendido no Hospital de Clínicas Veterinária da Universidade Federal de Pelotas (UFPEL), apresentando emagrecimento progressivo, episódios de diarreia hemorrágica e prolapso retal. A avaliação clínica evidenciou mucosas pálidas, desidratação leve e dor abdominal. Os exames laboratoriais evidenciaram hematócrito $30 \%$ e a ecografia abdominal revelou linfonodos mesentéricos aumentados. 
O paciente foi hospitalizado para estabilização hídrica, sedação para redução do prolapso retal, teve alta hospitalar com diagnóstico inicial de gastroenterite e prescrição constituída por vermífugo, sulfametoxazol, metronidazol, meloxicam, tramadol, dipirona em doses usuais por 7 dias e dieta orientada. Após 15 dias do primeiro atendimento, retornou com recidiva do prolapso, mucosas pálidas, linfonodos submandibulares reativos e relato do proprietário que o tratamento não havia sido realizado conforme prescrito. Foi solicitada nova ecografia abdominal a qual revelou intussuscepção em segmento de alça intestinal caudal ao rim esquerdo e presença de massa de contorno bem definido, medindo aproximadamente $6 \times 4 \mathrm{~cm}$ em abdômen esquerdo, sugestivo de neoplasia (Figura 1).

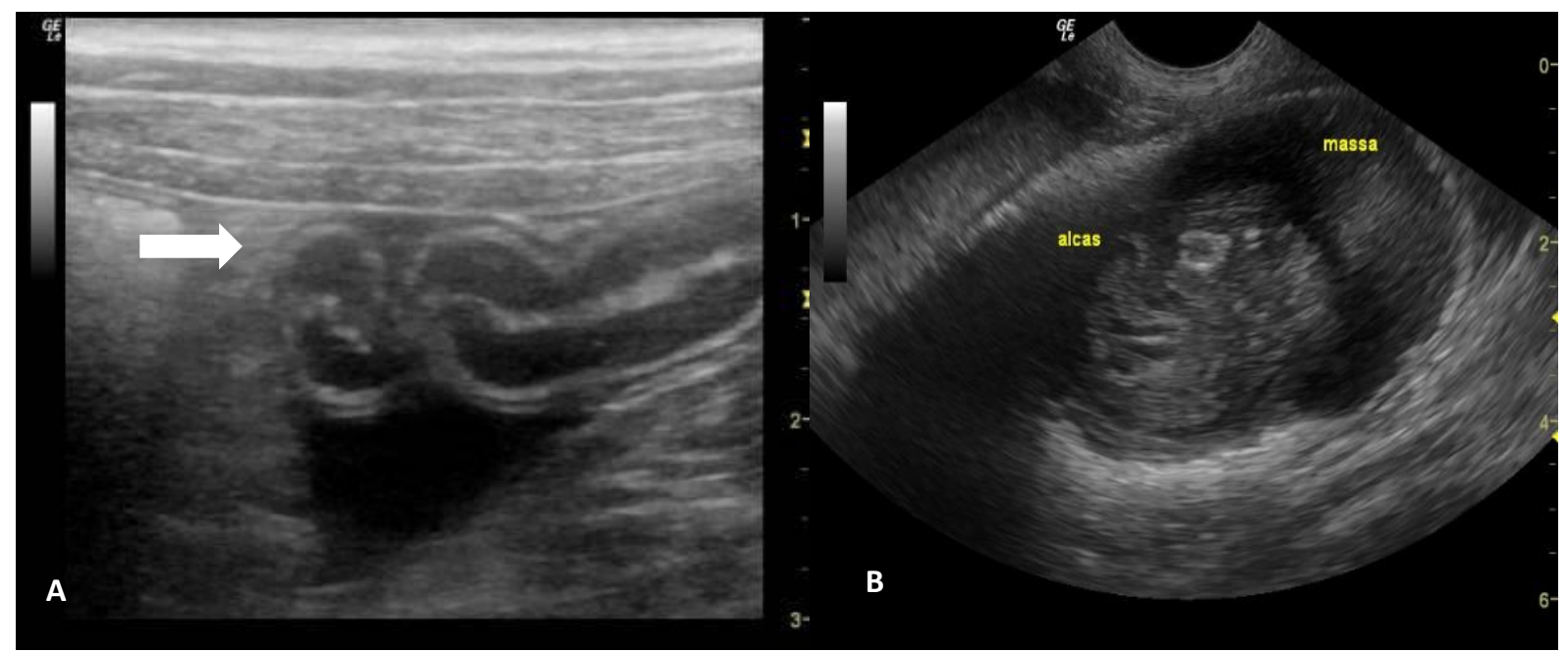

Figura 1. Imagem de ecografia abdominal de um cão com linfoma alimentar, revelando intussuscepção de alça intestinal (Aseta) e massa tumoral de contorno bem definido, medindo aproximadamente $6 \times 4 \mathrm{~cm}$ em abdômen esquerdo (B).

Após estabilizado, o animal foi encaminhado ao setor cirúrgico para enterectomia parcial do cólon descendente com ressecção da massa (Figura 2) compreendendo margem de $2 \mathrm{~cm}$ cranial e caudal ao tumor, redução da intussuscepção e anastomose término-terminal, na sequência a celiorrafia ocorreu de rotina. O paciente foi mantido hospitalizado com medicamentos analgésicos (tramadol, dipirona, meloxicam), protetor gástrico (ranitidina) e antibióticos (sulfametoxazol e metronidazol) em doses usuais, teve alta no terceiro dia do pós-operatório com manutenção das medicações prescritas por quatro dias e dieta controlada.
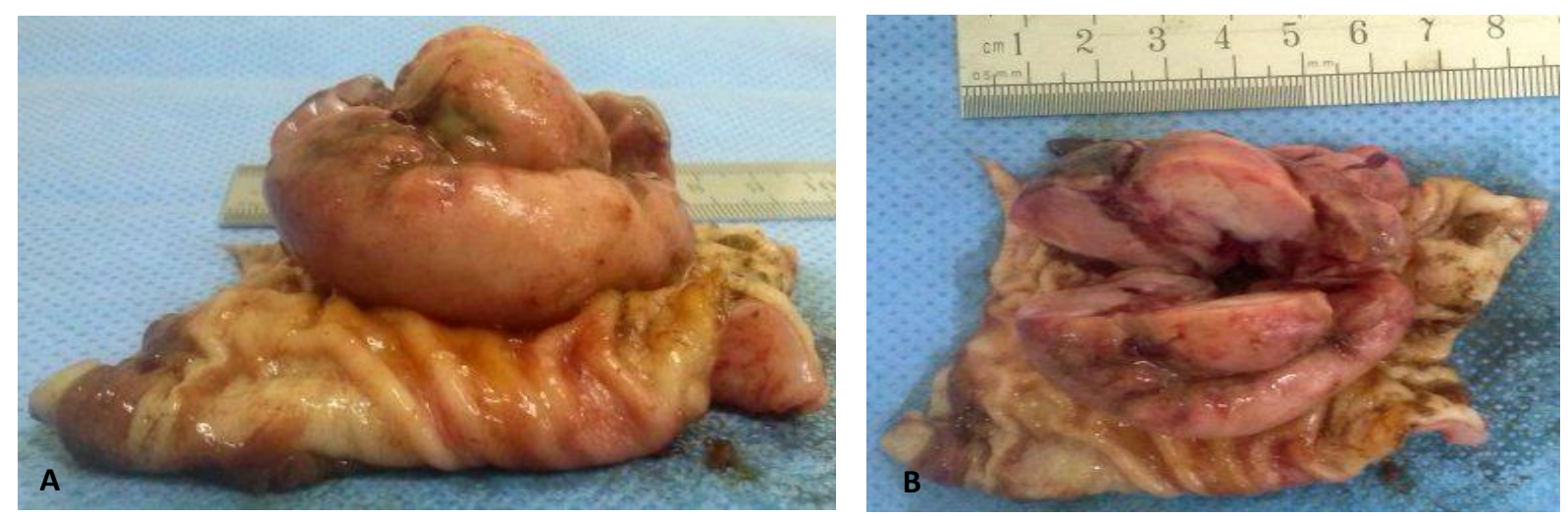

Figura 2. Imagem de um segmento do colón descendente após enterectomia em um cão jovem, que apresentava constipação, intussuscepção e prolapso retal. (A) Massa tumoral transmural projetada para a luz intestinal. (B) Massa tumoral após o corte sagital revelando aspecto maciço.

A massa foi encaminhada para o setor de patologia e o diagnóstico histopatológico revelou linfoma intestinal com proliferação de células linfoides nas camadas mucosa, submucosa e muscular e serosa do cólon descendente, com acentuado pleomorfismo e necrose, constituído de linfócitos pleomórficos, com núcleo grande, um ou mais nucléolos evidentes e citoplasma eosinofílico (Figura 3). 
Foi preconizado um protocolo quimioterápico, entretanto o tutor não aceitou a terapia. Dez dias após a cirurgia, o animal retornou ao hospital veterinário apresentando-se discretamente apático e com desconforto à palpação abdominal. Fez-se terapia de suporte com tramadol $(5 \mathrm{mg} / \mathrm{kg} / \mathrm{TID})$, prednisona $(1 \mathrm{mg} / \mathrm{kg} / \mathrm{BID})$ e enrofloxacina $(5 \mathrm{mg} / \mathrm{kg} / \mathrm{SID})$. Ao retorno em 30 dias, apresentava acentuação dos sinais clínicos e nova ecografia abdominal evidenciou massas disseminadas no abdômen envolvendo fígado, rins e linfonodos mesentéricos. Os tutores optaram pela eutanásia dois meses após o procedimento cirúrgico.
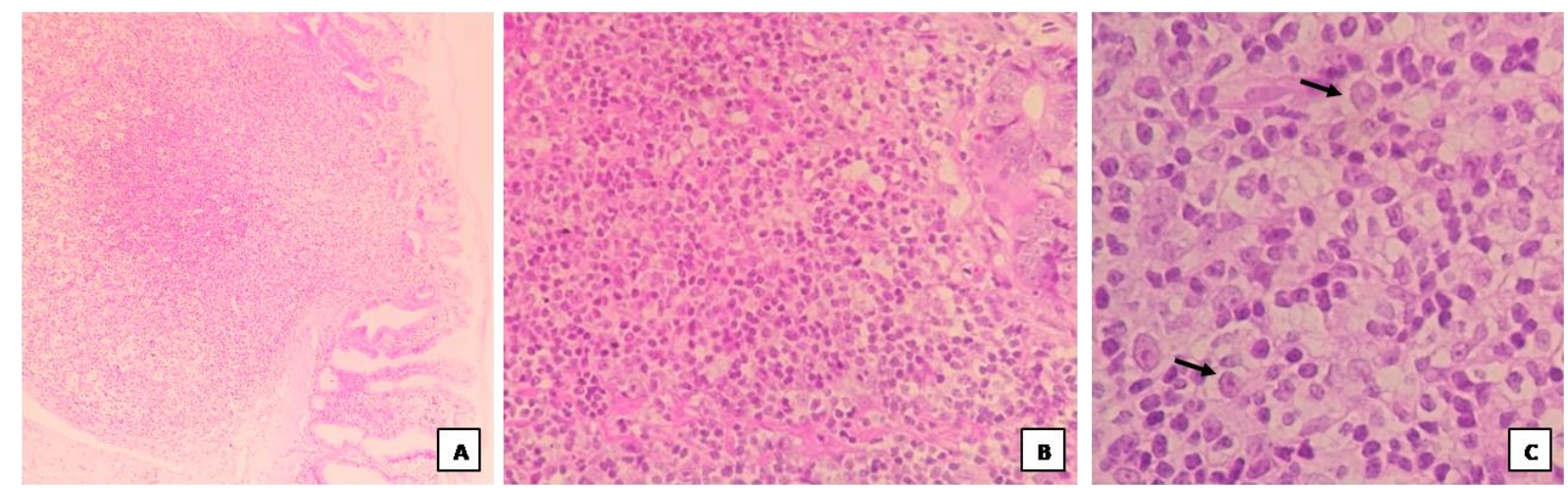

Figura 3. Avaliação histopatológica de uma massa intestinal de um cão. (A) Proliferação de células neoplásicas linfoides infiltrativas invadindo as camadas mucosa, submucosa, muscular e serosa do intestino, HE, obj. 4x. (B) Células neoplásicas, redondas, núcleos grandes, com um ou mais nucléolo evidente e citoplasma eosinofílico, HE, obj. 40x. (C) Marcado pleomorfismo nuclear dos linfócitos neoplásicos (setas) HE, obj. 100x.

\section{Resultados e discussão}

Segundo Fighera et al. (2006), a maior prevalência do linfoma alimentar é em cães de idade mediana a idosos, diferindo do presente relato, em que se tratava de um animal de quatro anos, e apenas $20 \%$ dos cães acometidos apresenta faixa etária inferior a quatro anos. Embora este paciente fosse SRD, os linfomas alimentares parecem ser mais prevalentes em Retrievers Dourados, Pastores Alemães, Boxers, Poodles, Basset Hounds e São Bernardos (Vail \& Ogilvie, 2003).

Assim como descrito por Cardoso et al. (2004), dentre os sinais clínicos iniciais havia emagrecimento, vômito e diarreia. O quadro clínico neste caso evoluiu para linfadenomegalia e anemia e segundo Vail et al. (2001), a linfadenomegalia é o sinal mais frequente e a anemia a alteração hematológica mais comum. O paciente apresentou prolapso retal e intussuscepção, e não foram encontrados relatos acerca dessas alterações decorrentes de linfoma alimentar em cães. Entretanto, Demetriou \& Welsh (1999) relataram prolapso retal decorrente de linfoma intestinal associado à intussuscepção em um felino.

O tratamento deve ser baseado em protocolos quimioterápicos, geralmente com excelente resposta, entretanto cães não tratados vivem entre quatro a seis semanas (Rodaski, 2008). Apesar deste dado, o paciente aqui descrito não foi submetido à quimioterapia por opção dos tutores e teve sobrevida de oito semanas; este tempo superior à literatura pode estar relacionado ao fato de o animal ser jovem, ter a única massa intestinal diagnosticada ressecada e ter recebido tratamento de suporte após o diagnóstico.

\section{Conclusão}

O linfoma alimentar é uma neoplasia de evolução rápida que, embora pouco comum, acomete também cães jovens, requer diagnóstico diferencial de enfermidades que cursam com gastroenterite e a sobrevida sem quimioterapia é curta, com progressão metastática acentuada.

\section{Referências bibliográficas}

Cardoso, M. J. L., Machado, L. H. A., Moutinho, F. Q. \& Padovani, C. R. (2004). Sinais clínicos do linfoma canino. Archives of Veterinary Science, 9(2):19-24.

Demetriou, J. L. \& Welsh, E. M. (1999). Rectal prolapse of an ileocaecal neoplasm associated with intussusception in a cat. Journal of Feline Medicine and Surgery, 1(4):253-256. 
Ettinger, S. N. (2003). Principles of treatment for canine lymphoma. Clinical Techniques in Small Animal Practice, 18(2):92-97.

Fighera, A. R. (2002). Linfossarcoma em cães. Ciência Rural, 32(5):895-899.

Fighera, R. A., Souza, T. M., Rodrigues, A. \& Barros, C. S. L. (2006). Aspectos clinicopatológicos de 43 casos de linfoma em cães. Revista Científica de Medicina Veterinária-Pequenos Animais e Animais de Estimação, 4(12):139-146.

Gieger, T. (2001). Alimentary lymphoma in cats and dogs. Veterinary Clinics of North America: Small Animal Practice, 41(2):419-432.

Lowe, D. A. (2004). Alimentary lymphosarcoma in a 4-year-old Labrador retriever. Canadian Veterinary Journal, 45610-612.

Rodaski, S. (2008). Quimioterapia antineoplásica em cães e gatos. São Paulo: MedVet Livros.

Sequeira, J. L., Franco, M., Bandarra, E. P., Figueiredo, L. M. A. \& Rocha, N. S. (1999). Características anatomoclínicas dos linfomas caninos na região de Botucatu, São Paulo. Arquivo Brasileiro de Medecina Veterinária e Zootecnia, 51(3):245-249.

Silva, F. E., Scofano, V. \& Ascoly, M. H. (2003). Fístula entero-vesical como complicação de linfoma intestinal: Relato de caso. Revista Brasileira de Coloproctologia, 23(3):200-204.

Vail, D. M., Macewen's \& Young, K. M. (2001). Canine lymphoma and lymphoid leukemias. In S. J. Witrow \& E. G. Macewen's (Eds.), Small animal clinical oncology. $3^{\circ} \mathrm{rd} / \mathrm{ed}$. Philadelphia: Saunders Company:558-590. (pp. 558-590). Philadelphia, USA: Saunders Company.

Vail, D. M. E. \& Ogilvie, G. K. (2003). Neoplasias linfoides. In S. J. Bichard \& R. G. Sherding (Eds.), Manual Saunders: Clínica de Pequenos Animais (pp. 227-233). São Paulo, Brasil: Roca.

Recebido: 9 de maio, 2019.

Aprovado: 2 de junho, 2019.

Publicado: 26 de junho, 2019.

Licenciamento: Este artigo é publicado na modalidade Acesso Aberto sob a licença Creative Commons Atribuição 4.0 (CC-BY 4.0), a qual permite uso irrestrito, distribuição, reprodução em qualquer meio, desde que o autor e a fonte sejam devidamente creditados 